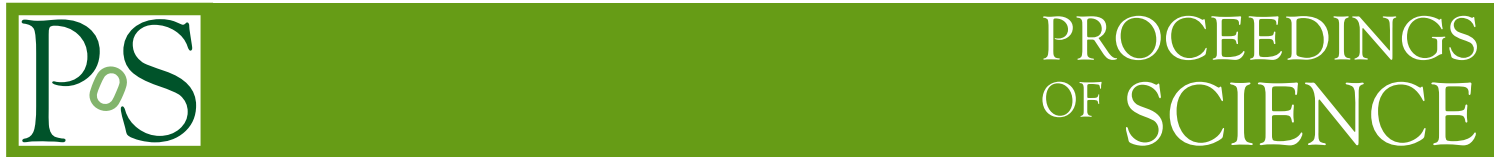

\title{
Improved heavy quark action
}

\author{
Norman H. Christ*, Huey-Wen Lin, Min Li \\ RBC and UKQCD Collaborations \\ Department of Physics, Columbia University, New York, NY 10027, USA \\ E-mail: nhcephys.columbia.edu, minxoleelphys.columbia.edu, \\ hwlin@phys.columbia.edu
}

\begin{abstract}
Building upon the work of the Fermilab and Tsukuba groups, we discuss improved lattice actions capable of describing onium and heavy-light systems with errors of order $(\vec{p} a)^{2}$ but accurate to arbitrary order in $(m a)^{n}$, where $a$ is the lattice spacing, $\vec{p}$ is the spatial momentum of the heavy quark(s) and $m$ the heavy quark mass. We demonstrate using both a tree-level example and an inductive proof that this accuracy can be achieved for hadronic masses using an action containing only three mass-dependent parameters: the bare quark mass $\left(m_{0}\right)$, an anisotropy coefficient $(\zeta)$ of the Dirac spatial derivative term and a coefficient $\left(c_{P}\right)$ of the chromomagnetic Pauli term. Further improvement terms are needed to calculate matrix elements involving spinor fields or composite operators. This represents a simplification of earlier results and makes practical the non-perturbative evaluation of these coefficients.
\end{abstract}

XXIVth International Symposium on Lattice Field Theory

July 23-28, 2006

Tucson, Arizona, USA

\footnotetext{
* Speaker.
} 


\section{Introduction.}

Lattice QCD is the clear method of choice to treat the non-perturbative effects of QCD. However, the charm and bottom quarks are sufficiently massive that the usual requirement that the particle masses are small compared to the inverse lattice spacing, $m \ll 1 / a$ is not satisfied for the lattice spacings presently accessible to full QCD lattice simulations. This is especially true for the more compute-intensive lattice fermion formulations where the light quarks are treated in a fashion respecting chiral symmetry (such as domain wall or overlap fermions).

Fortunately, for many important questions it is possible to avoid the difficulties associated with an overly large value of $m a$ by using a low-energy effective theory to describe the massive quarks in which the effects of the large mass are treated approximately (heavy quark effective theory), where the quarks are treated as non-relativistic (NRQCD) or where the errors of order $(m a)^{n}$ are compensated by appropriate mass-dependent coefficients in an effective Lagrangian (Fermilab or relativistic heavy quark (RHQ) method).

In this talk we will focus on the RHQ method developed by the Fermilab group [1] and further explored by the group at Tsukuba [2]. This offers the advantage of being able to treat a range of quark masses including both the $m \ll 1 / a$ and $1 / a \ll m$ limits and thus being consistent with an eventual continuum limit with $m \ll 1 / a$. In addition, this approach permits a straight-forward use of non-perturbative techniques, avoiding reliance on lattice perturbation theory. The results presented here summarize the work reported in Refs [3] and [4]. Further non-perturbative results can be found in the contribution of Huey-Wen Lin to this conference [5].

\section{Symanzik improvement applied to heavy quarks.}

We begin with the standard lattice action for Wilson fermions:

$$
S_{\mathrm{lat}}=\sum_{n^{\prime}, n} \bar{\psi}_{n^{\prime}}\left(\gamma^{\mu} D^{\mu}+m_{0}-\frac{r}{2}\left(D^{\mu}\right)^{2}+\sum_{i, j} \frac{i}{4} c_{S W} \sigma_{\mu \nu} F_{\mu v}\right)_{n^{\prime}, n} \psi_{n},
$$

where

$$
\begin{aligned}
\left(D_{\mu} \psi\right)_{n}= & \frac{1}{2}\left[U_{\mu}(n) \psi_{n+\hat{\mu}}-U_{\mu}(n-\hat{\mu})^{\dagger} \psi_{n-\hat{\mu}}\right] \\
\left(D_{\mu}^{2} \psi\right)_{n}= & {\left[U_{\mu}(n) \psi_{n+\hat{\mu}}+U_{\mu}(n-\hat{\mu})^{\dagger} \psi_{n-\hat{\mu}}-2 \psi_{n}\right] } \\
\left(F_{\mu \nu} \psi\right)_{n}= & \frac{1}{8} \sum_{s, s^{\prime}= \pm 1} s s^{\prime}\left[U_{s \mu}(n) U_{s^{\prime} v}(n+s \hat{\mu})\right. \\
& \left.\times U_{-s \mu}\left(n+s \hat{\mu}+s^{\prime} \hat{v}\right) U_{-s^{\prime} v}\left(n+s^{\prime} \hat{v}\right)-\text { h.c. }\right] \psi_{n} .
\end{aligned}
$$

The long-distance, on-shell properties of this theory can be described, including errors of order $a$, by the continuum Symanzik effective Lagrangian:

$$
S_{\mathrm{eff}}=\int d^{4} x \bar{\psi}(x)\left(\gamma^{\mu} D^{\mu}+m_{r}-a \frac{r^{c}}{2}\left(D^{\mu}\right)^{2}+a \sum_{\mu, v} \frac{i}{4} c_{S W}^{c} \sigma_{\mu \nu} F_{\mu \nu}\right) \psi(x) .
$$

In the usual Symanzik improvement program, one then argues that the unwanted coefficient $r^{c}$ can be removed by a redefinition of the fields $\psi$ and $\bar{\psi}$ while the second coefficient $c_{S W}^{c}$ can be set to 
zero by appropriately adjusting the parameters in the lattice Lagrangian in Eq. 2.1 (in this case only $m_{0}$ and $c_{S W}$ need be tuned) [6].

El-Khadra, Kronfeld and Mackenzie argued that this approach could describe a system including heavy quarks even in the case $m a \sim 1$ provided one works in the heavy quark rest system, incorporates the resulting loss of axis interchange symmetry and uses improvement coefficients which depend on $m a$.

We will now discuss this approach in detail. Begin by working in the heavy quark rest system and assuming that $|\vec{p} a| \ll 1$. Allow for the possibility that ma may be of order 1 by including terms in the continuum, effective Lagrangian which are of arbitrarily high order in $m a$ and $D_{0} a$. Thus, we use:

$$
\mathscr{L}_{\text {eff }}=\mathscr{L}_{\text {eff },-1}+\mathscr{L}_{\text {eff }, 0}+\mathscr{L}_{\text {eff }, 1}+\ldots
$$

where

$$
\begin{aligned}
\mathscr{L}_{\mathrm{eff},-1}= & \bar{\psi}\left(\frac{1}{a} B^{-1,1}+\gamma^{0} D^{0} C^{-1,1}\right) \psi \\
\mathscr{L}_{\mathrm{eff}, 0}= & \bar{\psi}\left(\left\{\vec{\gamma} \vec{D}, B^{0,1}\right\}+a\left\{\left[\vec{\gamma} \vec{D}, \gamma^{0} D^{0}\right], C^{0,1}\right\}\right) \psi \\
\mathscr{L}_{\mathrm{eff}, 1}= & a \bar{\psi}\left(\vec{D}^{2} B^{1,1}+a\left\{\vec{D}^{2}, \gamma^{0} D^{0}\right\} C^{1,1}\right. \\
& +\left[\gamma^{i}, \gamma^{j}\right]\left[D^{i}, D^{j}\right] B^{1,2}+a\left\{\left[\gamma^{i}, \gamma^{j}\right]\left[D^{i}, D^{j}\right], \gamma^{0} D^{0}\right\} C^{1,2} \\
& \left.+\left[\gamma^{i}, \gamma^{0}\right]\left[D^{i}, D^{0}\right] B^{1,3}+a\left[\left[\gamma^{i}, \gamma^{0}\right]\left[D^{i}, D^{0}\right], \gamma^{0} D^{0}\right] C^{1,3}\right) \psi
\end{aligned}
$$

The expansion described in Eq. 2.6 is in powers of $|\vec{p} a|$. The quantities $m a$ and $D_{0} a$ are treated as of order 1 and included to all powers in each of the terms $\mathscr{L}_{\text {eff,-1}}, \mathscr{L}_{\text {eff }, 0}$, etc. Thus, the leading terms, given in Eq. 2.7, contain factors of $m_{0}$ and $\gamma^{0} D_{0}$ and are therefore labeled as order $a^{-1}$. The coefficients $B^{i, j}$ and $C^{i, j}$ which appear in Eqs. 2.7-2.9 are real polynomials of arbitrary order in $m_{0} a,\left(D_{0} a\right)^{2}$ and $g^{2}$. We must now determine how general a lattice action must be used so that, with a proper choice of the coefficients in this lattice action, the equivalent continuum action will agree through order $|\vec{p} a|$ and to arbitrary order in $m_{0} a$ with the usual continuum Dirac action.

\section{On-shell improvement.}

The first step in determining the form of such an lattice action is to recognize which of the unwanted terms in Eq. 2.6 can be simply removed by a redefinition of the continuum fields $\psi(x)$ and $\bar{\psi}(x)$. With the exception of possible non-Lorentz covariant terms associated with spinor fields discussed below, the choice of field operator should have no effect on the resulting on-shell Green's functions. Thus, we are free to make arbitrary, non-singular field transformations to simplify the form of the continuum effective Lagrangian given in Eqs. 2.6-2.9. (This is equivalent to using the equations of motion to simplify Eqs. 2.6-2.9.)

We therefore consider a series of field transformations involving terms of increasing order in the expansion parameter $|\vec{p} a|$ :

$$
O(1): \quad \psi=\left(1+R^{0,1}+a \gamma^{0} D^{0} S^{0,1}\right) \psi^{\prime}
$$




$$
\begin{aligned}
& \bar{\psi}=\bar{\psi}^{\prime}\left(1+R^{0,1}-a \gamma^{0} \overleftarrow{D}^{0} S^{0,1}\right) \\
& O(a): \quad \psi=\psi^{\prime}\left(1+a \vec{\gamma} \vec{D} R^{1,1}+a\left[\vec{\gamma} \vec{D}, a \gamma^{0} D^{0}\right] S^{1,1}\right) \psi^{\prime} \\
& \bar{\psi}=\bar{\psi}^{\prime}\left(1-a R^{1,1} \vec{\gamma} \overleftarrow{D}-a S^{1,1}\left[\vec{\gamma} \overleftarrow{D}, a \gamma^{0} \overleftarrow{D}^{0}\right]\right) \\
& O\left(a^{2}\right): \quad \psi=\left(1+a^{2} \vec{D}^{2} R^{2,1}+a^{2}\left\{\vec{D}^{2}, a \gamma^{0} D^{0}\right\} S^{2,1}\right. \\
& +a^{2}\left[\gamma^{i}, \gamma^{j}\right]\left[D^{i}, D^{j}\right] R^{2,2}+a^{2}\left\{\left[\gamma^{i}, \gamma^{j}\right]\left[D^{i}, D^{j}\right], a \gamma^{0} D^{0}\right\} S^{2,2} \\
& \left.+a^{2}\left[\gamma^{i}, \gamma^{0}\right]\left[D^{i}, D^{0}\right] R^{2,3}+a^{2}\left[\left[\gamma^{i}, \gamma^{0}\right]\left[D^{i}, D^{0}\right], a \gamma^{0} D^{0}\right] S^{2,3}\right) \psi^{\prime} \\
& \bar{\psi}=\bar{\psi}^{\prime}\left(1+a^{2} \overleftarrow{D}^{2} R^{2,1}-a^{2}\left\{\overleftarrow{D}^{2}, a \gamma^{0} \overleftarrow{D}^{0}\right\} S^{2,1}\right. \\
& +a^{2}\left[\gamma^{i}, \gamma^{j}\right]\left[\overleftarrow{D}^{i}, \overleftarrow{D}^{j}\right] R^{2,2}-a^{2}\left\{\left[\gamma^{i}, \gamma^{j}\right]\left[\overleftarrow{D}^{i}, \overleftarrow{D}^{j}\right], a \gamma^{0} \overleftarrow{D}^{0}\right\} S^{2,2} \\
& \left.+a^{2}\left[\gamma^{i}, \gamma^{0}\right]\left[\overleftarrow{D}^{i}, \overleftarrow{D}^{0}\right] R^{2,3}+a^{2}\left[\left[\gamma^{i}, \gamma^{0}\right]\left[\overleftarrow{D}^{i}, \overleftarrow{D}^{0}\right], a \gamma^{0} \overleftarrow{D}^{0}\right] S^{2,3}\right)
\end{aligned}
$$

As is discussed in detail in Ref. [3], the real coefficients $R^{i, j}$ and $S^{i, j}$ appearing in these transformations can be chosen to significantly simply the continuum effective Lagrangian in Eqs. 2.6-2.9. Our basic strategy is to linearize the problem by working to a fixed order $N=k+l+n$ in an expansion in term of order $\left(m_{0} a\right)^{k}\left(D_{0} a\right)^{2 l} g^{2 n}$. We can then perform induction in $N$. The resulting effective continuum action can then be simplified to the form:

$$
S_{\mathrm{eff}}=\int d^{4} x \bar{\psi}(x)\left(\gamma^{0} D^{0}+\zeta^{c} \vec{\gamma} \cdot \vec{D}+m_{r}+\sum_{\mu, v} \frac{i}{4} c_{P}^{c} a \sigma_{\mu \nu} F_{\mu \nu}\right) \psi(x) .
$$

This implies that a continuum result, accurate through order $|\vec{p} a|$ and to arbitrary order in $m_{0} a$ can be obtained from a lattice action with only three adjustable parameters:

$$
S_{\mathrm{lat}}=\sum_{n^{\prime}, n} \bar{\psi}_{n^{\prime}}\left(\gamma^{0} D^{0}+\zeta \vec{\gamma} \cdot \vec{D}+m_{0}-\frac{1}{2}\left(D^{0}\right)^{2}-\frac{\zeta}{2} \vec{D}^{2}+\sum_{\mu, v} \frac{i}{4} c_{P} \sigma_{\mu v} F_{\mu v}\right)_{n^{\prime}, n} \psi_{n} .
$$

Thus, we need only fix the bare quark mass $m_{0}$, the anisotropy parameter $\zeta$ and the coefficient $c_{P}$ of the chromomagnetic or Pauli term so that the parameters in the corresponding continuum action of Eq. 3.7 take their relativistic form, in particular $\zeta^{c}=c_{P}^{c}=0$.

This is a somewhat surprising result because in the earlier Fermilab and Tsukuba papers the need to separately tune the coefficients $c_{B}$ and $c_{E}$ of the $\bar{\psi} \sigma^{i, j} F^{i, j} \psi$ and $\bar{\psi} \sigma^{0, j} F^{0, j} \psi$ terms is anticipated. However, by performing the order $a^{2}$ transformation in Eqs. 3.5 and 3.6 on the terms in the original Lagrangian of order $1 / a$ (Eq. 2.7) we can remove the difference between $c_{B}$ and $c_{E}$. This can be seen schematically by performing such a transformation with parameter $\alpha$ :

$$
\begin{aligned}
\mathscr{L}_{\text {eff }}= & \bar{\psi}(x)\left(1+\alpha a^{2}\left[\gamma^{i}, \gamma^{0}\right]\left[D^{i}, D^{0}\right]\right) \cdot\left(\gamma^{0} D^{0}+m_{r}\right)\left(1+\alpha a^{2}\left[\gamma^{i}, \gamma^{0}\right]\left[D^{i}, D^{0}\right]\right) \psi(x) \\
= & \bar{\psi}(x)\left(\gamma^{0} D^{0}+m_{r}+\alpha 2 m_{r} a^{2}\left[\gamma^{i}, \gamma^{0}\right]\left[D^{i}, D^{0}\right]\right. \\
& \left.+\alpha a^{2}\left\{\left[\gamma^{i}, \gamma^{0}\right]\left[D^{i}, D^{0}\right], \gamma^{0} D^{0}\right\}\right) \psi(x) .
\end{aligned}
$$

The resulting term with coefficient $\alpha 2 m_{r} a^{2}$ can be used to set the coefficient $c_{E}$ term equal to that of the $c_{B}$ term without any tuning of the lattice Lagrangian. (The extra factor of $m_{r} a$ present in 
this coefficient does not cause a problem because the mass-independent part of the coefficients of $\bar{\psi} \sigma^{i, j} F^{i, j} \psi$ and $\bar{\psi} \sigma^{0, j} F^{0, j} \psi$ will be axis interchange symmetric and hence equal.) Note the extra $\sim \alpha a^{2}\left[\left[D^{i}, D^{0}\right], D^{0}\right]$ generated by this transformation is explicitly of order $|\vec{p} a|^{2}$ and can be dropped.

If we tried to change the coefficient of $\bar{\psi} \sigma^{i, j} F^{i, j} \psi$ by making a similar $\left(1+\beta \bar{\psi} \sigma^{i, j} F^{i, j} \psi\right)$ transformation the extra term would be of order $a$ and could not be dropped.

\section{On-shell spinor matrix elements.}

In a relativistic theory, field transformations have no effect on the on-shell Green's functions beyond the introduction of multiplicative "wave function" renormalization factors. This implies that if a field transformation exists which will remove unwanted terms in the Lagrangian, this transformation need not be actually made because the original, untransformed fields will yield the same on-shell Green's functions. It is only in this case where these transformation need not be actually carried out that the parameters which they remove (and upon which these transformations then depend) can be ignored.

The actual effect of such non-linear field transformations can be seen concretely by examining a Green's functions containing the transformed field but evaluated in the original theory. As shown in Fig. 1 the pole that appears when the transformed field is Fourier transformed and $p^{2}$ allowed to approach $m_{r}^{2}$ will arise only in the class of graphs which are one-particle-reducible with respect to this transformed field operator. The entire external momentum $p^{\mu}$ must flow through a single line if that diagram is have a pole. The effect of using the proper, transformed field is described by the factor corresponding to the right-hand-side of Fig. 1.

For a scalar or relativistic spinor field, this corresponds to a simple multiplicative constant. However, in the non-relativistic situation that results from working in the heavy quark rest system, a spinor Z-factor can occur:

$$
\psi_{\alpha}(x)=Z_{\alpha, \beta} \psi_{\beta}^{\prime}(x)=\frac{1}{Z_{q}}\left(1-i \delta_{\psi} \vec{\gamma} \cdot \vec{\nabla}\right)_{\alpha, \beta} \psi_{\beta}^{\prime}(x)
$$

This implies that correct relativistic Green's functions will be obtained only after the spinor transformation described in Eq. 4.1 has been performed. (This conclusion has been emphasized by the Tsukuba group and adds to their count of needed parameters.) As is suggested by the notation, the parameter $\delta_{\psi}$ will depend on the particular fermion field that is being used. While one value of $\delta_{\psi}$ is required for a perturbative calculation, quite a different one will be needed for the interpolating, composite field needed to create or destroy a charmed baryon. Fortunately, $\delta_{\psi}$ is relatively easy to determine non-perturbatively by simply examining the long-distance spinor structure of the corresponding fermion propagator.

\section{Tree-level check.}

As a check of these conclusions we have carried out a tree-level calculation of the quark propagator, including its on-shell spinor structure, as well as the spatial and temporal components 


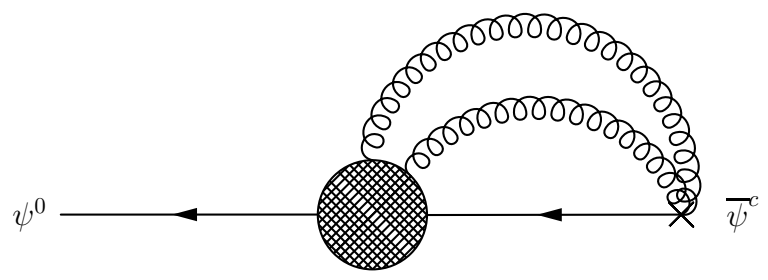

Figure 1: A class of diagrams contributing to the $4 \times 4$ spinor renormalization matrix $\bar{Z}_{\alpha \beta}$ connecting the improved and un-improved fields, $\bar{\psi}^{c}$ and $\bar{\psi}^{0}$ respectively. Here the point-like vertex represented by the cross corresponds to the composite, improved operator $\psi^{c}$ which contains products of the quark and gluon fields. The graph contained within the shaded circle must be one-particle-irreducible.

of the quark-gluon vertex, again on the quark mass-shell. Beginning with the general anisotropic lattice Wilson action,

$$
\begin{aligned}
S_{\text {lat }}= & \sum_{n^{\prime}, n} \bar{\psi}_{n^{\prime}}\left(\gamma^{0} D^{0}+\zeta \vec{\gamma} \cdot \vec{D}+m_{0}-\frac{r_{t}}{2}\left(D^{0}\right)^{2}-\frac{r_{s}}{2} \vec{D}^{2}\right. \\
& \left.+\sum_{i, j} \frac{i}{4} c_{B} \sigma_{i j} F_{i j}+\sum_{i} \frac{i}{2} c_{E} \sigma_{0 i} F_{0 i}\right)_{n^{\prime}, n} \psi_{n},
\end{aligned}
$$

we found that the following choices for $m_{r} a, Z_{q}, \zeta$ and $\delta_{\psi}$ gave continuum results through order $|\vec{p} a|$ for any value of $m_{0} a$ in the range $0<m_{0}<\infty$ :

$$
\begin{aligned}
m_{r} a & =\ln \left(\frac{m_{0} a+r_{t}+\sqrt{\left(m_{0} a\right)^{2}+2 r_{t} m_{0} a+1}}{1+r_{t}}\right) \\
Z_{q} & =\cosh \left(m_{r} a\right)+r_{t} \sinh \left(m_{r} a\right) \\
\zeta^{2} & =\frac{\sinh \left(m_{r} a\right)}{m_{r} a}\left(r_{t} \sinh \left(m_{r} a\right)+\cosh \left(m_{r} a\right)\right)-r_{s} \sinh \left(m_{r} a\right) \\
\delta_{\psi} & =\frac{\zeta}{2 \sinh \left(m_{r} a\right)}-\frac{1}{2 m_{r} a} .
\end{aligned}
$$

This is the conclusion expected from our earlier discussion. The details of this calculation are given in Ref. [3].

\section{Conclusions.}

As we have seen, it is possible to describe the physics of an onium or heavy-light system accurately through order $|\vec{p} a|$ and to all orders in $m a$ if three parameters are properly tuned. The first parameter, the bare quark mass $m_{0}$, must be determined from experiment and is ultimately tuned non-perturbatively to make the resulting spectrum agree with reality. The second, anisotropy parameter $\zeta$ can be easily determined from the momentum-dependence of the energy of one of the particles being studied. It can be tuned, again non-perturbatively, to insure that $E(\vec{p})=m_{r}+\frac{\vec{p}^{2}}{2 m_{r}}$. The final Pauli parameter, $c_{P}$ is more difficult to determine. Given the small number of parameters, it is quite straight-forward to determine this from experiment also, adjusting it to fit the spinspin splitting of an onium or heavy-light meson. Of course, one then loses an interesting lattice 
prediction. Alternatively, $c_{P}$ can be computed from first principles, by employing a step-scaling method which begins with a sufficiently small lattice spacing (and correspondly small physical volume) that a standard relativistic treatment for the heavy quark is accurate. One then considers a series of increasing physical volumes and lattice spacings, matched to maintain constant finitevolume physics. Both approaches are quite practical, even with dynamical fermions as discussed in Refs. [4] and [5].

This approach can be applied to systems involving either charm or bottom quarks (or both). However, since $\vec{p} \propto \alpha_{s} m$ for onium systems our method will be more accurate for heavy-light systems where $\vec{p} \propto \Lambda_{\mathrm{QCD}}$. Similarly we expect greater accuracy for charmonium that bottomonium. Since no assumption is made regarding the relative size of $m$ and $a$, the continuum, $a \rightarrow 0$ limit can be taken. However, unless $m a$ can be neglected, one expects the coefficient of the neglected $(\vec{p} a)^{2}$ term to itself be a function of $m a$ which will modify the usual $a^{2}$ approach to the continuum limit.

While the results presented here are based on a theoretical analysis, the idea that the removal of order $|\vec{p} a|$ errors could be achieved without tuning $c_{E}$ was suggested to us by the numerical studies described in Ref. [4].

\section{Acknowledgments}

We acknowledge helpful discussions with Tanmoy Bhattacharya, Peter Boyle, Paul Mackenzie, Andreas Kronfeld, Sinya Aoki, Yoshinobu Kuramashi and members of the RBC collaboration. In addition, we thank Peter Boyle, Dong Chen, Mike Clark, Saul Cohen, Calin Cristian, Zhihua Dong, Alan Gara, Andrew Jackson, Balint Joo, Chulwoo Jung, Richard Kenway, Changhoan Kim, Ludmila Levkova, Xiaodong Liao, Guofeng Liu, Robert Mawhinney Shigemi Ohta, Konstantin Petrov, Tilo Wettig and Azusa Yamaguchi for developing with us the QCDOC machine and its software. This development and the resulting computer equipment used in this calculation were funded by the U.S. DOE grant DE-FG02-92ER40699, PPARC JIF grant PPA/J/S/1998/00756 and by RIKEN. This work was supported by DOE grant DE-FG02-92ER40699 and we thank RIKEN, Brookhaven National Laboratory and the U.S. Department of Energy for providing the facilities essential for the completion of this work.

\section{References}

[1] A. X. El-Khadra, A. S. Kronfeld, and P. B. Mackenzie, Massive fermions in lattice gauge theory, Phys. Rev. D55 (1997) 3933-3957, [hep-lat/9604004].

[2] S. Aoki, Y. Kuramashi, and S.-i. Tominaga, Relativistic heavy quarks on the lattice, Prog. Theor. Phys. 109 (2003) 383-413, [hep-lat/0107009].

[3] N. H. Christ, M. Li, and H.-W. Lin, Relativistic heavy quark effective action, hep-lat/0608006.

[4] H.-W. Lin and N. Christ, Non-perturbatively determined relativistic heavy quark action, hep-lat/0608005.

[5] H.-W. Lin, Charmed spectroscopy from a nonperturbatively determined relativistic heavy quark action in full qcd, PoS LAT2006 (2006) 184.

[6] B. Sheikholeslami and R. Wohlert, Improved continuum limit lattice action for qcd with wilson fermions, Nucl. Phys. B259 (1985) 572. 